\title{
ESTABILIDADE OXIDATIVA DO ÓLEO LUBRIFICANTE CONTAMINADO POR GASOLINA
}

\author{
Maria Carolina da S. Coelho ${ }^{1.2}$, Roberta M. Teixeira ${ }^{1}$, Sérgio L. C. Viscardi ${ }^{1}$, Jo Dweck ${ }^{2}$ \\ ${ }^{1}$ Ipiranga Produtos de Petróleo S.A., Rio de Janeiro, Brasil \\ ${ }^{2}$ Escola de Química, Universidade Federal do Rio de Janeiro.
}

E-mail: carolinasc@ipiranga.com.br, robertamt@ipiranga.com.br, viscardi@ipiranga.com.br, jodweck@yahoo.com.br

\section{RESUMO}

Durante a operação do motor ocorre a contaminação do óleo lubrificante pela passagem do combustível através dos pistões para o cárter. Este fato acelera a degradação do óleo lubrificante e a sua oxidação, diminuindo sua vida útil. Quanto ao uso de lubrificantes em motores automotivos, um dos maiores problemas é a deterioração provocada por esta contaminação pelo combustível utilizado, seja gasolina C (gasolina e etanol anidro) ou etanol hidratado, que é o caso do Brasil. Os novos motores do tipo flex também devem ser considerados, em que misturas de álcool - gasolina $\mathrm{C}$ pode ser usado em proporções diferentes. O comportamento termo-oxidativo do óleo lubrificante contaminado com gasolina de até $6 \% \mathrm{v} / \mathrm{v}$, foi estudado usando o método ASTM D2272 - Testes de Oxidação em Vaso de Pressão Rotativo (RPVOT) e pelo Método de Teste Padrão ASTM D6186 - 08 - Tempo de indução à oxidação por pressão diferencial calorimétrica (PDSC). Ponto de Fulgor (ASTM D93), as análises também foram realizadas. Os resultados indicaram que com o aumento da contaminação de gasolina, o tempo de indução à oxidação das reduções de óleo lubrificante, que por sua vez, promovem um efeito significativo sobre a redução do ponto de fulgor da contaminação do lubrificante.

Palavras-chave: Óleo lubrificante, gasolina, contaminação, RPVOT, PDSC, ponto de Fulgor.

\section{INTRODUÇÃO}

A todo o momento somos surpreendidos pela evolução tecnológica e os impactos que dela decorrem. Nas indústrias esta evolução pode ser percebida pela utilização de máquinas cada vez mais sofisticadas para atender a um mercado cada vez mais exigente e competitivo. A lubrificação é fundamental para que máquinas com partes móveis funcionem de uma forma mais regular e econômica, permitindo assim uma vida mais longa e útil e o bom funcionamento a fim de evitar paradas no processo e maiores custos operacionais. Os lubrificantes formam um filme fino de óleo sobre as superfícies metálicas, evitando contatos diretos entre as mesmas, que aplicado às máquinas, cria uma camada impermeável entre as peças, reduzindo seu aquecimento e evitando que aquelas em movimento se desgastem indevidamente por ação do atrito. 
Assim como as máquinas, os lubrificantes sofreram alterações tecnológicas para atender as necessidades extremas em processos industriais e a evolução do setor automotivo. A escolha de um lubrificante correto deve ser feita para que se obtenha um processamento com mínimo de problemas decorrentes de atrito. Entretanto, sua troca requer alguns cuidados. A compatibilidade, composição química, polaridade e interações com outros aditivos ou até mesmo outros lubrificantes, tudo deve ser considerado em relação à formulação escolhida [1].

A estabilidade de um lubrificante pode ser afetada pelo ambiente no qual ele está em operação, fatores externos podem influenciar diretamente no seu desempenho, tais como contaminação, temperatura, oxidação e meios ácidos limitam sua vida útil. [2].

Óleos lubrificantes podem sofrer dois tipos de degradação [3]: degradação oxidativa que conduz o lubrificante a uma polimerização inicial, que resulta na formação de lodo e verniz (os insolúveis oxigenados); e degradação térmica que também pode formar verniz de carbono insolúveis quando causada pela absorção de ar, ou depósitos de materiais carbonáceos, quando causadas por superfícies metálicas quentes ou por descargas eletrostáticas.

Alguns métodos de análise foram desenvolvidos para estudos das alterações nas propriedades físico-químicas de lubrificantes e outros combustíveis líquidos que ocorrem por aquecimento [4]. Entre eles, para avaliar a resistência os testes de oxidação acelerada em vasos de pressão (RPVOT, RBOT, PETROXY) são os mais usados [5-8].

Os dois primeiros métodos têm sido mais utilizados e são baseados no tempo necessário para uma queda de pressão de oxigênio fixa que ocorre durante a sua ação oxidante no óleo. Já o último método é baseado no tempo necessário para deter a queda de pressão do oxigênio, o que também indica o tempo completo da oxidação [8].

Um dos métodos padrão para avaliar a estabilidade térmica oxidativa é a norma ASTM D6186 - 08, que mede o tempo de indução de oxidação (OIT) de óleos lubrificantes por calorimetria exploratória diferencial de alta pressão PDSC [9]. Este método mede o OIT de óleos lubrificantes submetidos à atmosfera de oxigênio a elevadas pressões e temperaturas entre $130{ }^{\circ} \mathrm{C}$ e $210^{\circ} \mathrm{C}$. O OIT é definido como o tempo decorrido a partir da primeira exposição à atmosfera oxidante até o tempo do início da temperatura extrapolada de início (onset) do pico exotérmico de oxidação.

O desenvolvimento da indústria automobilística tem causado aumentos significativos na produção de gasolina automotiva nos últimos anos. A gasolina é um líquido inflamável e volátil composto por uma mistura complexa de hidrocarbonetos que podem ser obtidos por destilação de petróleo [10]. Sua produção pode também ser decorrente de craqueamento de sub-produtos de refino de petróleo.

No Brasil, a gasolina do tipo $\mathrm{C}$ que é utilizada é uma mistura de etanol anidro, numa proporção de $25 \%$ em volume deste álcool, de acordo com a Agência Nacional de Petróleo, Gás Natural e Biocombustíveis (ANP) da resolução nº 40/2013 [11].

O desenvolvimento da indústria automobilística refletiu na produção da gasolina automotiva, provocando seu aumento significativo nos últimos anos. $\mathrm{O}$ presente trabalho visa contribuir com utilização dessas técnicas, no estudo de possíveis efeitos deletérios a lubrificantes por contaminações com gasolina quando do uso em motores movidos a este combustível. 


\section{MATERIAIS E MÉTODOS}

Para este estudo foi utilizada uma amostra comercial de óleo lubrificante semissintético SAE 10W30, e uma amostra de gasolina C, esta preparada em laboratório a partir de mistura de etanol anidro e gasolina A comercial, na proporção de $25 \%$ em volume de álcool, Agência Nacional do Petróleo, Gás Natural e Biocombustíveis (ANP) Resolução $\mathrm{n}^{\mathrm{o}}$ 40/2013[11]. As misturas de gasolina $\mathrm{C}$ em óleo lubrificante foram preparadas contendo as seguintes concentrações: $(0,0 ; 2,0 ; 4,0$ e 6,0$) \%$ v/v, para os testes de RPVOT e PDSC, já para os testes de Ponto de Fulgor foram usadas as seguintes concentrações: $(0,5 ; 1,0 ; 1,5 ; 2,0 ; 4,0$ e 6,0$) \% \mathrm{v} / \mathrm{v}$. Todos os frascos âmbar onde as misturas foram guardadas até a análise, foram preenchidos sem "headspace" e mantidos acondicionados em um freezer à temperatura de $(9 \pm 1)^{\circ} \mathrm{C}$.

Tabela - 1 Especificação do Óleo Lubrificante - SAE 10W30

LUBRIFICANTE - SAE 10W30

\begin{tabular}{ccccc}
\hline Ensaio & Especificação & Método & Resultado & Unidade \\
\hline Aparência Visual & Límpido & Próprio & Límpido & - \\
Verificação da Cor & L2.0- L4.0 & ASTM D1500 & L2.0 & - \\
Teste de Água & Ausente & Próprio & Ausente & - \\
Teste de Densidade à 20/4 ${ }^{\circ} \mathrm{C}$ & $0,8300-0,8900$ & ASTM D4052 & $\mathbf{0 , 8 6 0 9}$ & $\mathrm{g} / \mathrm{cm}^{3}$ \\
Teste de Viscosidade $100^{\circ} \mathrm{C}$ & $9,30-12,50$ & SAE J300/ASTM D445 & $\mathbf{1 0 , 7 2}$ & $\mathrm{mm}^{2} / \mathrm{s}$ \\
Teste de Viscosidade $40^{\circ} \mathrm{C}$ & $60,0-90,0$ & ASTM D445 & $\mathbf{6 9 , 6 3}$ & $\mathrm{mm}^{2} / \mathrm{s}$ \\
Teste de Índice de Viscosidade & $140-160$ & ASTM D2270 & $\mathbf{1 4 3}$ & $\mathrm{IV}$ \\
Teste de Ponto de Fulgor ${ }^{\circ} \mathrm{C}$ & $200-300$ & ASTM D92 & $\mathbf{2 4 4}$ & ${ }^{\circ} \mathrm{C}$ \\
Teste de Ponto de Flú́dez ${ }^{\circ} \mathrm{C}$ & $(-42)-(-39)$ & ASTM D97 & $\mathbf{- 3 6}$ & ${ }^{\circ} \mathrm{C}$ \\
CCS -25 ${ }^{\circ} \mathrm{C}$ & $0-7000$ & SAE J300 & $\mathbf{5 9 9 8}$ & $\mathrm{Cp}$ \\
Perda por Evaporação - Noack & $0-15$ & API SL & $\mathbf{1 0 , 0 7}$ & $\%$ \\
Teor de Cálcio & $0,161-0,197$ & ASTM D4628 & $\mathbf{0 , 1 6 1}$ & $\%$ \\
Teor de Zinco & $0,0815-0,0996$ & ASTM D4628 & $\mathbf{0 , 0 8 2}$ & $\%$ \\
Teste de Espuma, Seq. I & $10-00$ & API SL/ASTM D892 & $\mathbf{0 / 0}$ & $\mathrm{mL}$ \\
Teste de Espuma, Seq. II & $50 / 00$ & API SL/ASTM D892 & $\mathbf{0 / 0}$ & $\mathrm{mL}$ \\
Teste de Espuma, Seq. III & $10-00$ & API SL/ASTM D892 & $\mathbf{0 / 0}$ & $\mathrm{mL}$ \\
MRV & $0-60.000$ & SAE J300 & $\mathbf{3 3 . 0 0 0}$ & $\mathrm{mPas}$ \\
HT/HS & 2.9 minimum & SAE J300 & $\mathbf{3 . 0 7 8}$ & $\mathrm{mPas}$ \\
\hline
\end{tabular}


Tabela - 2 Especificação da Gasolina Tipo C

GASOLINA TIPO C

\begin{tabular}{ccccc}
\hline Ensaio & Especificação & Método & Resultado & Unidade \\
\hline Aspecto & Límpido e isento de impurezas & VISUAL & Límpido & - \\
Verificação da Cor & De incolor a amarelada & VISUAL & Amarelada & - \\
Etanol Anidro Combustível - EAC & 24 a 26 & NBR 13992 & $\mathbf{2 5}$ & $\%$ vol \\
Massa Específica a $20{ }^{\circ} \mathrm{C}$ & Anotar & ASTM D4052 & $\mathbf{7 5 0 , 3}$ & $\mathrm{Kg} / \mathrm{m}^{3}$ \\
Destilação & - & - & - & - \\
$10 \%$ Recuperados & máx 65 & ASTM D86 & $\mathbf{5 8 , 5}$ & ${ }^{\circ} \mathrm{C}$ \\
$50 \%$ Recuperados & máx 80 & ASTM D86 & $\mathbf{7 3 , 3}$ & ${ }^{\circ} \mathrm{C}$ \\
$90 \%$ Recuperados & máx 190 & ASTM D86 & $\mathbf{1 6 2 , 3}$ & ${ }^{\circ} \mathrm{C}$ \\
Ponto Final de Ebulição & máx 215 & ASTM D86 & $\mathbf{1 8 0 , 5}$ & ${ }^{\circ} \mathrm{C}$ \\
Resíduo & máx 2 & ASTM D86 & $\mathbf{1 , 3}$ & $\%$ vol \\
\hline
\end{tabular}

As análises do Tempo de Indução de Oxidação em Vaso de Pressão Rotativo (RPVOT) do óleo e das misturas foram realizadas pelo método ASTM D2272 [12], utilizando o equipamento Quantum Oxidation Tester, Tannas Co. Foram utilizados tubos de PVC com altura de aproximadamente $41 \mathrm{~mm}$, e diâmetro externo de $48 \mathrm{~mm}$ para preparação do fio de cobre em espiral. Cerca de $(55,6 \pm 0,3) \mathrm{g}$ de fio de cobre em espiral foram usados em cada análise como catalisador de oxidação introduzido em misturas de $(50 \pm 0,5) \mathrm{g}$ de amostra e $5 \mathrm{~mL}$ de água destilada. As condições experimentais utilizadas para todas as amostras foram: aquecimento desde temperatura ambiente de $25^{\circ} \mathrm{C}$ até $150{ }^{\circ} \mathrm{C}$, utilizando 90 psi de Oxigênio para pressurização inicial do vaso de pressão. A seguir, o tempo de indução de oxidação da amostra é determinado quando a pressão máxima atingida no aquecimento até $150{ }^{\circ} \mathrm{C}$, decresce 25 psi.

As análises de ponto de fulgor do óleo lubrificante e das misturas foram realizadas pelo método ASTM D93 [13], utilizando o equipamento Pensky-Martens Closed Cup Test, da Tanaka modelo APM-7.

Os testes de PDSC das amostras foram realizados em equipamento da TA Instruments, modelo Q2000, pelo método ASTM D6186 - 08 [9]. Foram utilizados cadinhos especiais de alumínio Tzero e tampas para selagem hermética com microfuro a laser, a fim de minimizar a volatilização de componentes da gasolina antes da análise e promover a melhor separação na perda de massa respectiva aos componentes de cada amostra durante as análises. Cerca de 5,00 mg de amostra foram utilizadas para as análises. Utilizou-se 100 psi de oxigênio para pressurização inicial da câmara de aquecimento do PDSC. A seguir depois de fechada a câmara, usou-se razão de aquecimento de $100^{\circ} \mathrm{C} \cdot \mathrm{min}^{-1}$ até $210{ }^{\circ} \mathrm{C}$ ficando 2 minutos a esta temperatura para estabilização da pressão. Após manteve-se condições isotérmicas por 150 minutos. Todos os gráficos foram obtidos utilizando o software Universal Analysis da TA Instruments.

\section{RESULTADOS E DISCUSSÃO}

\subsection{Testes de Oxidação em Vaso de Pressão Rotativo (RPVOT)}


Foram feitos Testes de Oxidação em Vaso de Pressão Rotativo (RPVOT), pelo método ASTM D2272 [12] com amostras de óleo lubrificante e misturas óleo lubrificante/gasolina, com foco na avaliação do efeito da contaminação. Nas Figuras 1 e 2 são mostradas curvas típicas obtidas para os casos do óleo lubrificante puro e para a mistura contendo $6 \%$ em volume de gasolina $\mathrm{C}$.

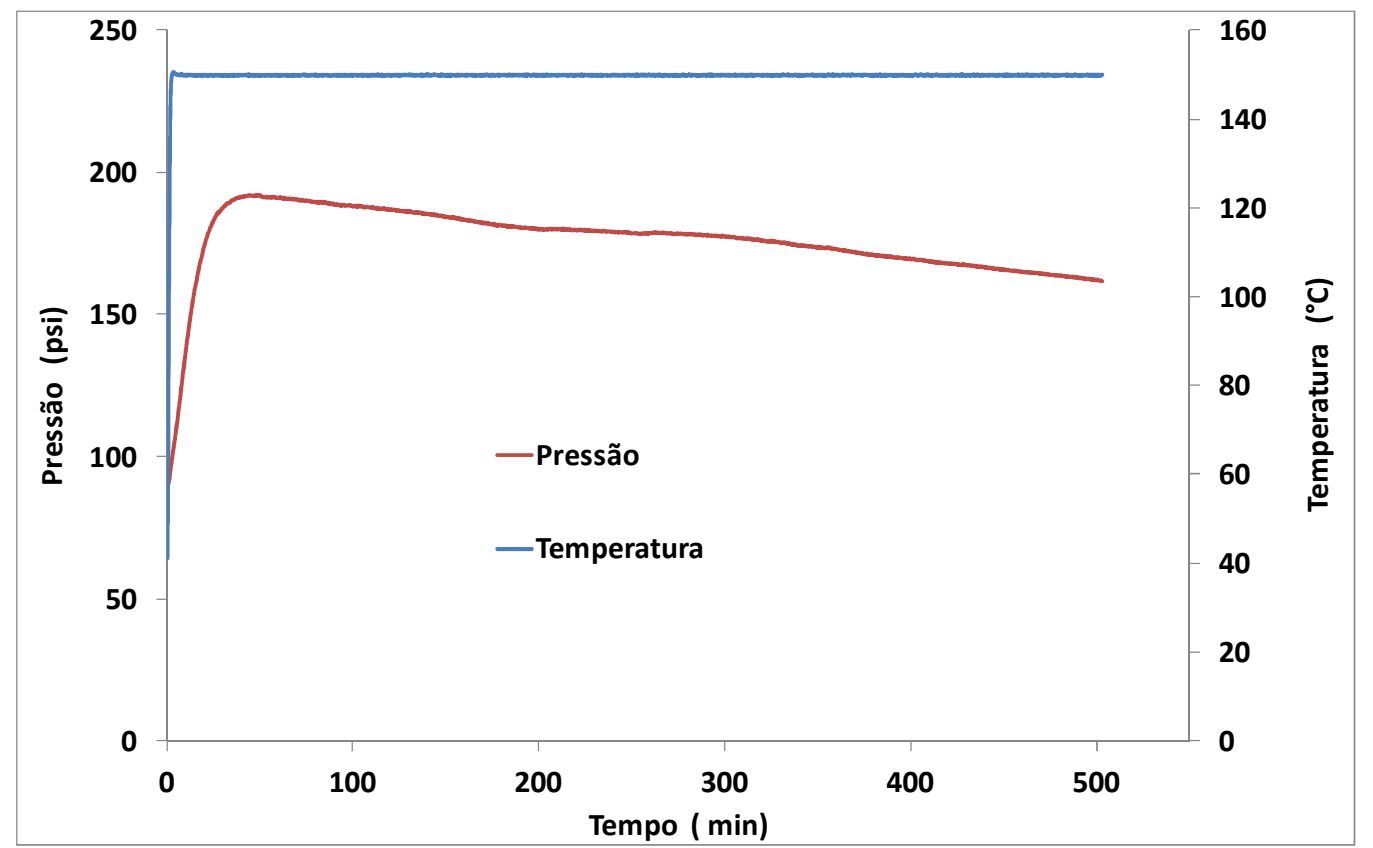

Figura - 1 Curva RPVOT do óleo lubrificante

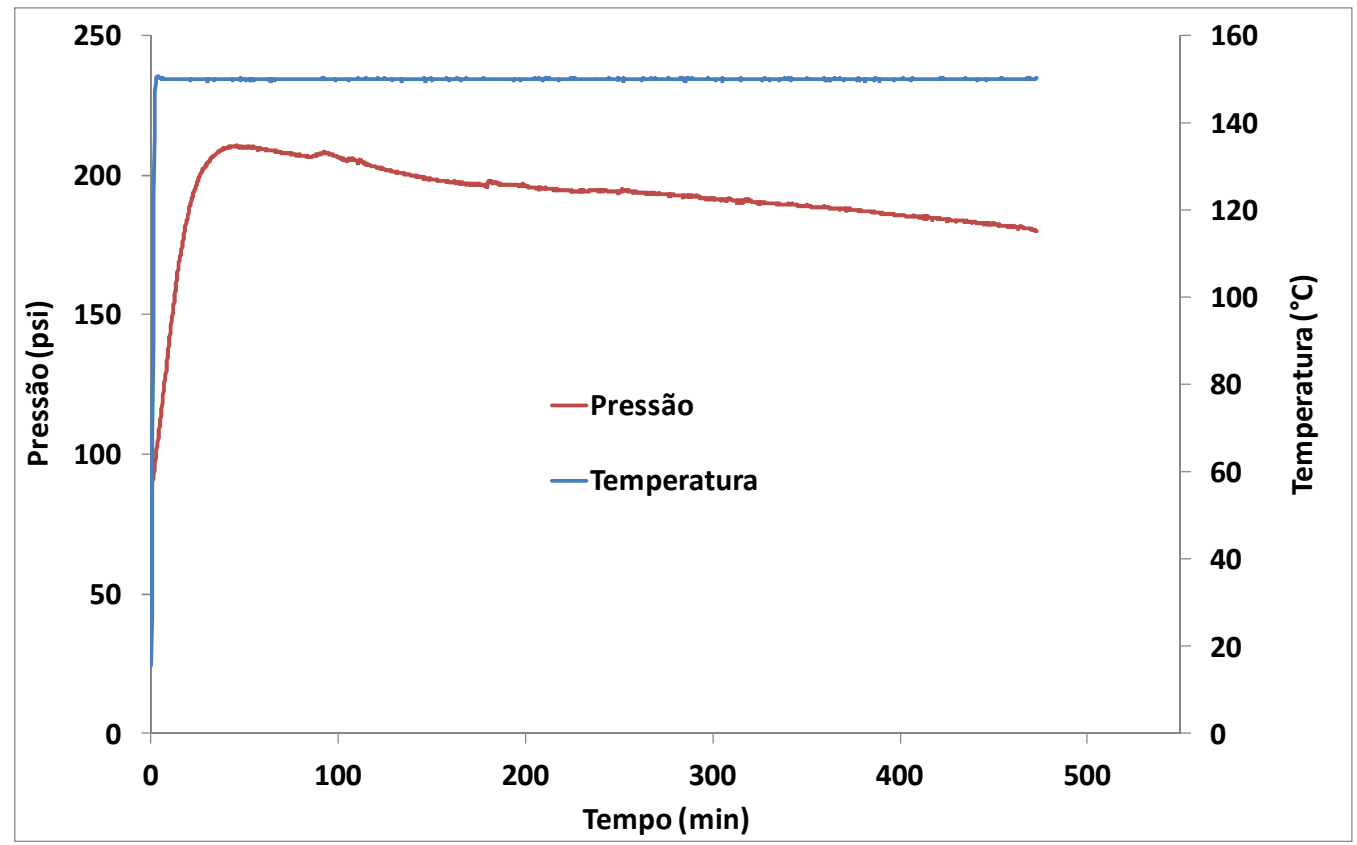

Figura - 2 Curva RPVOT da mistura óleo lubrificante e gasolina 6 \% v/v

Observa-se nas Figuras 1 e 2 que na presença de gasolina C, após aquecer a mistura até $150{ }^{\circ} \mathrm{C}$, a pressão máxima inicial atingida é maior, visto a volatilização de parte de seus componentes mais voláteis. 
A Tabela 3 mostra os tempos de indução de oxidação obtidos por RPVOT em duplicata e sua média. Esses valores representam o tempo de decréscimo de pressão de 25 psi, em relação à pressão máxima inicial em cada caso. A Figura 3, que apresenta a correlação obtida para os valores médios de tempo de indução de cada caso em função do teor de gasolina $\mathrm{C}$ na mistura, mostra que a mesma é uma função polinomial do segundo grau, indicando que quanto maior o teor de gasolina presente no óleo lubrificante mais rápido será seu processo de oxidação. Cabe notar que o coeficiente de correlação obtido é excelente, indicando que de fato, essa função representa o efeito da presença da gasolina no processo de oxidação do óleo lubrificante, nas condições do ensaio.

Tabela - 3 Resultados do tempo de indução de oxidação (TI) por RPVOT

\begin{tabular}{ccccc}
\hline \% de gasolina C & $\mathbf{0}$ & $\mathbf{2}$ & $\mathbf{4}$ & $\mathbf{6}$ \\
TI 1 (min) & 479 & 444 & 407 & 404 \\
TI 2 (min) & 436 & 450 & 415 & 307 \\
TI médio (min) & $\mathbf{4 5 8}$ & $\mathbf{4 4 7}$ & $\mathbf{4 1 1}$ & $\mathbf{3 5 6}$ \\
\hline
\end{tabular}

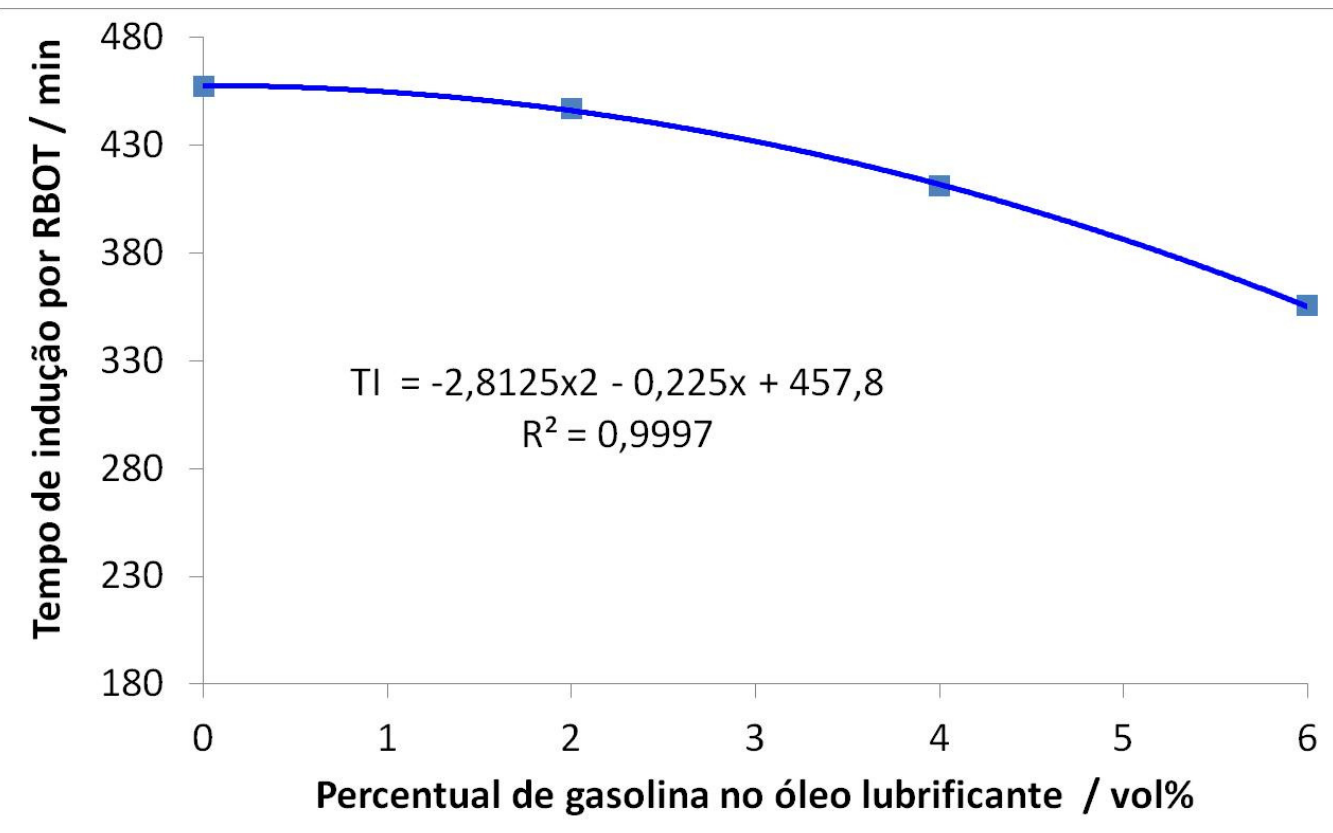

Figura - 3 Curva do tempo de indução de oxidação de misturas óleo lubrificante/ gasolina $\mathrm{C}$ em função do percentual de gasolina

\subsection{Ponto de fulgor}

Quando da realização dos ensaios observou-se que para concentrações maiores que $2 \% \mathrm{v} / \mathrm{v}$ de gasolina na mistura com óleo lubrificante, o ponto de fulgor era praticamente o mesmo que a $2 \%$, o que indicava a elevada volatilização dos componentes da gasolina à pressão ambiente. Além disso, esses resultados não eram representativos de fato do ponto de fulgor, pois teriam sido até menores, se a temperatura ambiente do laboratório fosse menor.

Cabe observar que em vista do ocorrido, embora as determinações de ponto de fulgor tivessem sido feitas inicialmente em vaso aberto para o óleo lubrificante, 
conforme recomenda a norma, elas tiveram que ser realizadas em vaso fechado para minimizar as perdas. Face a isto a avaliação da presença de gasolina no ponto de fulgor das misturas foi feita em concentrações até $2 \%$ de gasolina $\mathrm{C}$.

Os resultados de ponto de fulgor em vaso aberto para o óleo lubrificante e em vaso fechado para o óleo lubrificante e para as misturas, realizados em triplicata, estão na Tabela 4. Os valores de ponto de fulgor médio em vaso fechado estão representados na Figura 4. Observa-se que o ponto de fulgor das misturas decresce segundo uma função de terceiro grau do teor de gasolina na faixa analisada, o que também explica o porquê do limite de detecção para no máximo de $2 \%$ em volume de gasolina na mistura.

Tabela - 4 Pontos de fulgor do óleo lubrificante e das misturas $\left({ }^{\circ} \mathrm{C}\right)$

\begin{tabular}{c|c|cccccccc}
\hline Tipo de Vaso & em vaso aberto & \multicolumn{10}{c}{ em vaso fechado } \\
\hline \% de gasolina C & $\mathbf{0}$ & $\mathbf{0}$ & $\mathbf{0 . 5}$ & $\mathbf{1}$ & $\mathbf{1 . 5}$ & $\mathbf{2}$ & $\mathbf{4}$ & $\mathbf{6}$ \\
PF-1 & 244 & 204 & 114 & 90 & 65 & 30 & nd & nd \\
PF-2 & 244 & 206 & 108 & 86 & 65 & 32 & nd & nd \\
PF-3 & 242 & 206 & 108 & 83 & 58 & 28 & nd & nd \\
PF médio & $\mathbf{2 4 3}$ & $\mathbf{2 0 5}$ & $\mathbf{1 1 0}$ & $\mathbf{8 6}$ & $\mathbf{6 3}$ & $\mathbf{3 0}$ & & \\
\hline
\end{tabular}

* nd - não determinado

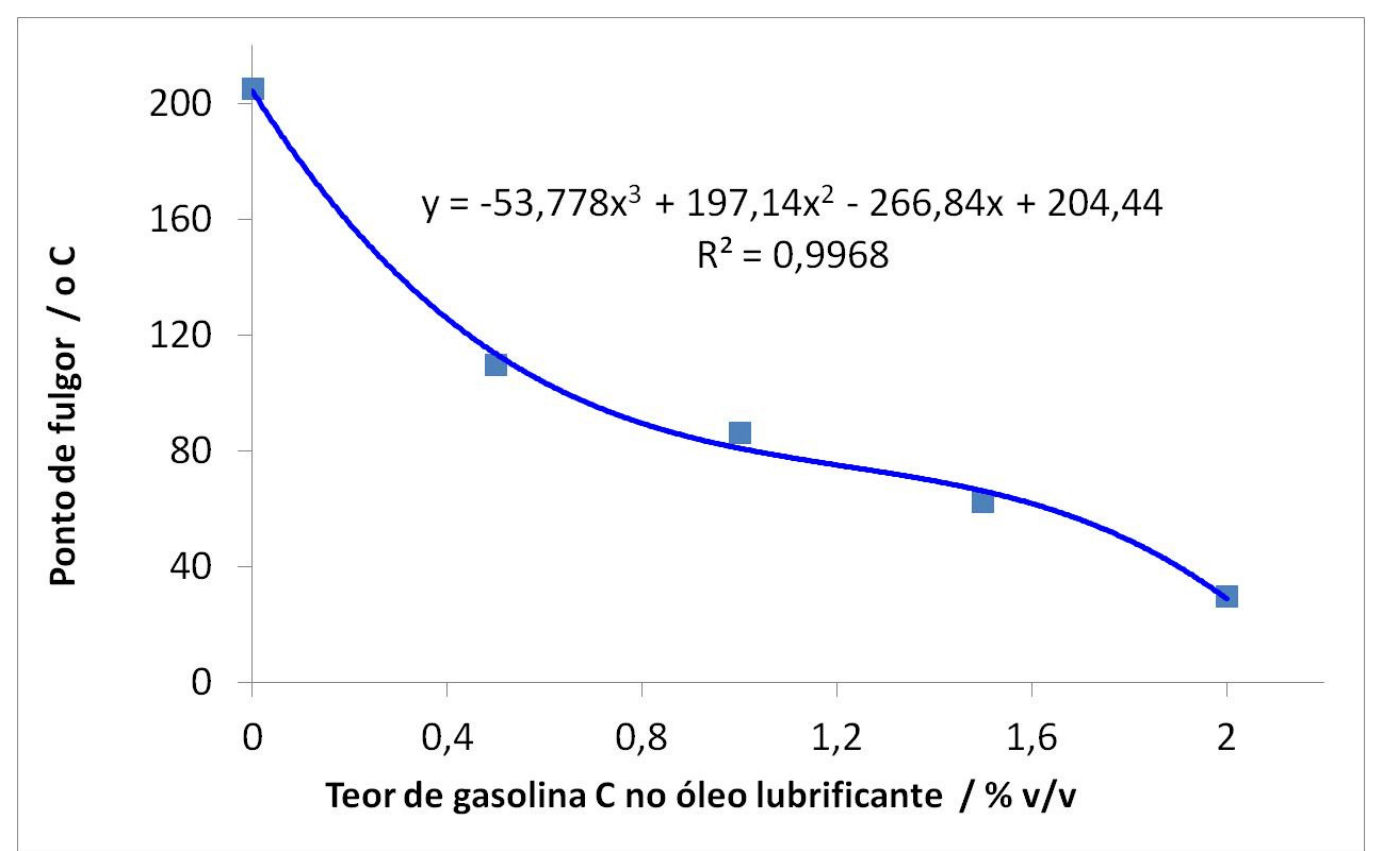

Figura - 4 Curva dos pontos de fulgor do óleo lubrificante e das misturas com gasolina $\mathrm{C}$

\subsection{Análises por PDSC}

A determinação das temperaturas de estabilidade termo-oxidativa do óleo lubrificante e da gasolina utilizados no presente estudo foram obtidas através das respectivas curvas PDSC, que estão mostradas nas Figuras 5 e 6.

Conforme se pode notar na Figura 5, à medida que se aumenta o teor de gasolina $\mathrm{C}$, diminui o tempo para se atingir o ponto máximo do pico PDSC de oxidação total das amostras, o que indica que a presença de gasolina acelera o processo de 
oxidação. Além disso, nota-se que a forma de oxidação para as duas maiores concentrações é diferente das anteriores (caso $2 \%$ e $0 \%$ ), ocorrendo as mesmas em menor tempo e maior intensidade, conforme indicado pelo formato mais agudo dos picos PDSC respectivos.

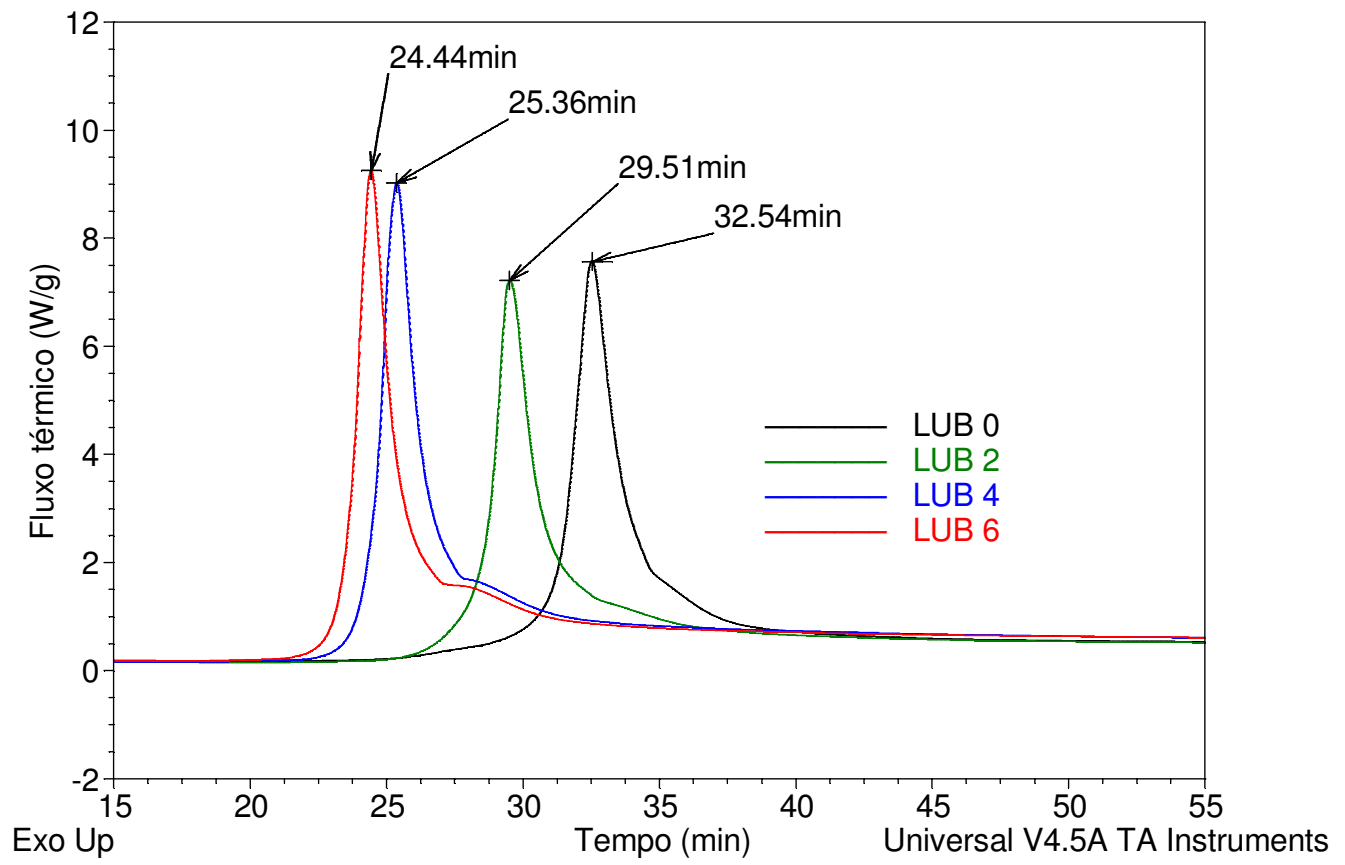

Figura - 5 Picos PDSC das amostras LUB 0 a LUB 6

A Figura 6 apresenta as mesmas curvas PDSC que a Figura 5, porém transladadas para poder melhor visualizar os tempos de indução de oxidação de cada caso, medidos pelos respectivos tempos de onset de cada pico PDSC. Pode-se ver que a maior concentração de gasolina $\mathrm{C}$ acelera significativamente $\mathrm{o}$ processo de oxidação do lubrificante em que esteja misturado.

Foram também calculadas e mostradas na Figura 6 as entalpias de oxidação total de cada caso. Observa-se que nos casos das duas maiores concentrações de gasolina $\mathrm{C}$, maiores energias totais são liberadas durante seu processo de oxidação, o que comprova as observações já feitas referentes às formas de oxidação mais intensas nesses casos. 


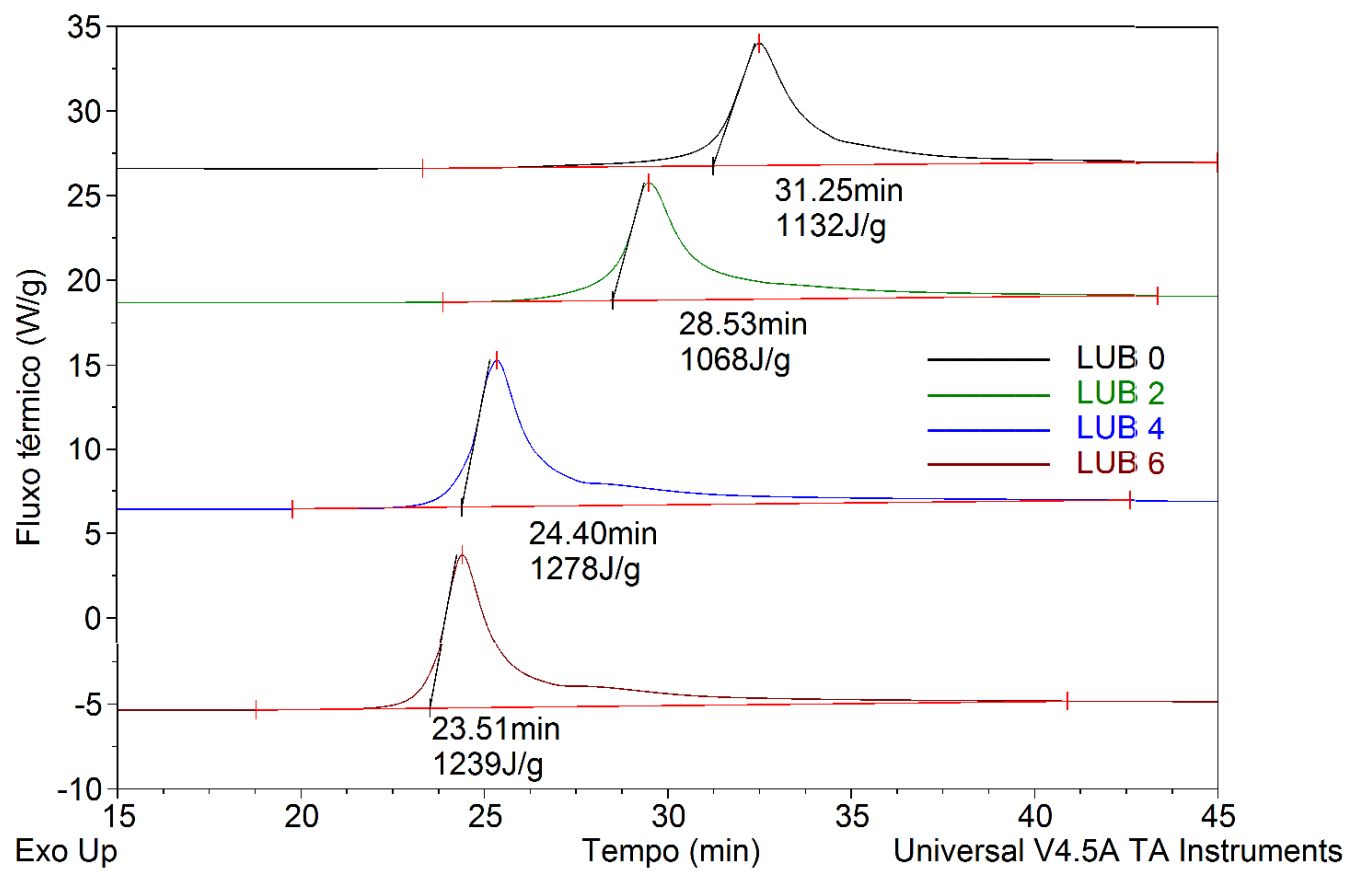

Figura - 6 Determinação dos tempos de indução de oxidação e das entalpias totais de oxidação das amostras analisadas.

Na Figura 7 observa-se que a diminuição do tempo de indução oxidativa do óleo lubrificante promovida pela presença de gasolina é uma função polinominal do segundo grau da concentração volumétrica de gasolina $\mathrm{C}$.

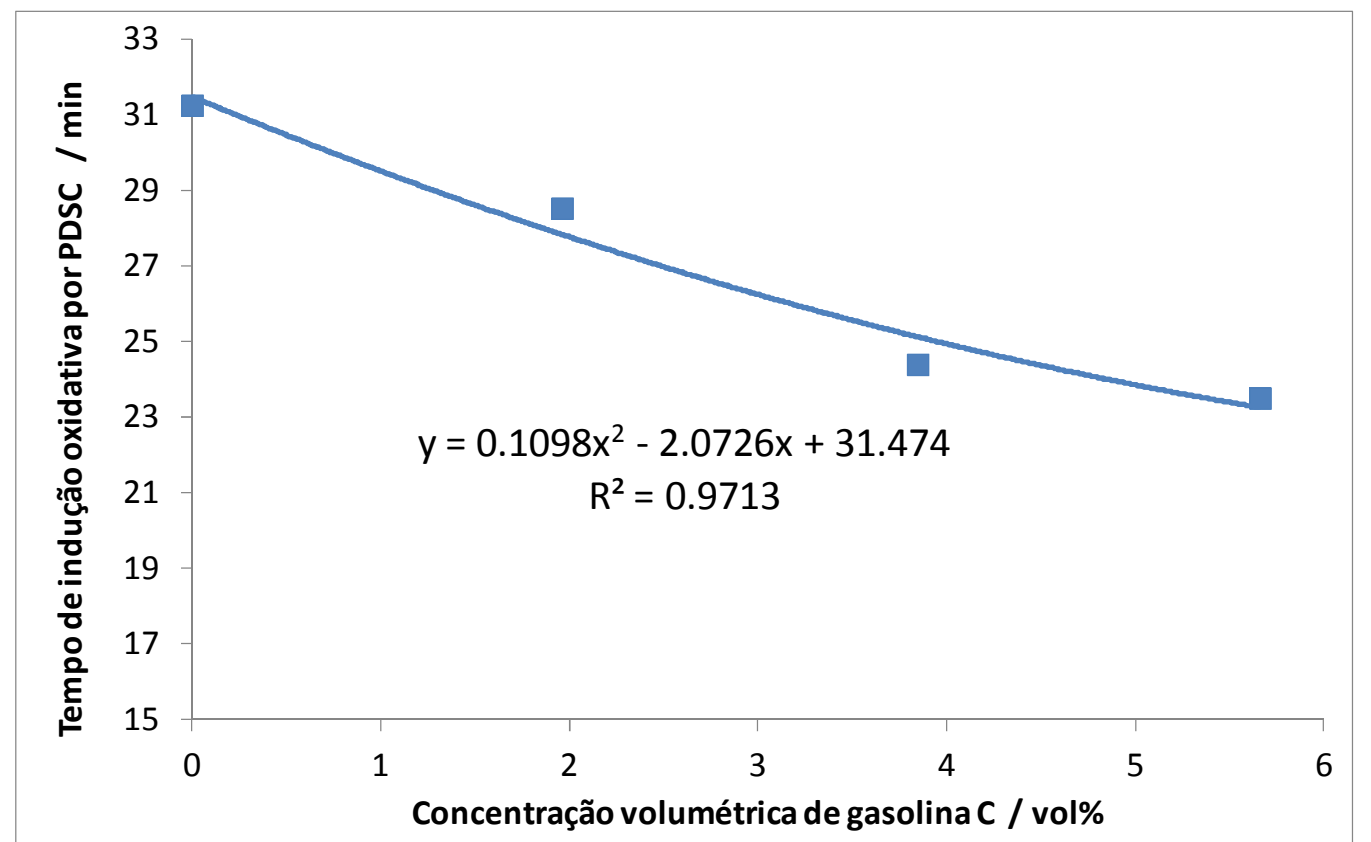

Figura - 7 Curva de tempo de indução de oxidação (OIT) do óleo lubrificante por PDSC, em função da concentração volumétrica presente de gasolina $\mathrm{C}$ 


\section{CONCLUSÕES}

Os testes de oxidação em vaso de pressão rotativo (RPVOT), que melhor simulam as condições operacionais do cárter, indicam que a presença da gasolina diminui o tempo de indução oxidativa do óleo lubrificante automotivo, indicando que esta presença é prejudicial à vida útil do óleo.

Essa diminuição do tempo de indução é uma função polinomial do segundo grau do teor de gasolina $\mathrm{C}$ presente no óleo lubrificante, indicando que à medida que a contaminação por gasolina aumenta, o processo de deterioração do óleo por oxidação aumenta significativamente.

Os resultados dos pontos de fulgor indicam que sistemas fechados mesmo em nível de pressão baixos possibilitam uma maior contaminação de óleo lubrificante por gasolina, enquanto que em sistemas abertos essa contaminação fica limitada, pela maior volatilização de componentes da gasolina nessas condições.

Por PDSC também se observa que, quando aumenta o teor de gasolina $\mathrm{C}$ presente no óleo lubrificante, seu processo de oxidação é acelerado, diminuindo o tempo de sua indução oxidativa de acordo com uma função polinominal do segundo grau da concentração volumétrica de gasolina $\mathrm{C}$, e em consequência, sua estabilidade oxidativa.

Verifica-se também que, quando elevados teores de gasolina estão presentes no óleo lubrificante automotivo, eles intensificam o seu processo de oxidação e promovem maior liberação de energia durante sua oxidação total.

Os resultados obtidos indicam que a estabilidade oxidativa de óleos lubrificantes contaminados por gasolina $\mathrm{C}$ pode ser avaliada tanto por testes de oxidação em vaso de pressão rotativo (RPVOT), assim como por calorimetria exploratória diferencial de alta pressão (PDSC).

\section{REFERÊNCIAS}

[1] RUPRECHT, V. C. Lubrificação, Fricção e Adesão. http: //www.uergs.edu.br, acesso em 16 de janeiro, 2013.

[2] CARRETEIRO, R. P., BELMIRO, P. N. A. Lubrificantes e Lubrificação Industrial $1^{\text {a }}$. Editora Interciência, Rio de Janeiro, p.69, 2006.

[3] WAGENSELLER, G Fluid analysis critical to maximizing lube-oil service life, Combined Cycle Journal, 4(2009)60-3

[4] HAINES, P.J., Principles of Thermal Analysis and Calorimetry - 2002, RSC Paperbacks.

[5] Serra, T Z, Cavalcanti, E J C, Sihvenger, J C, Mora, N D, Análise comparativa do desempenho do óleo lubrificante tipo turbina antes e após regeneração.

http://www.foz.unioeste.br/ lamat/publicoleolub/oleolubcbm2007.pdf, acessado em 12 de outubro, 2014.

[6] CAVAlCANTI, E. J. C, MORA, N.D, SERRA, T, Z, Estudo do Desempenho do Óleo Lubrificante em Pontos Diferentes da Turbina. 
http://www.foz.unioeste.br/ lamat/publicoleolub/oleolubc3n2007.pdf, acessado em 12 de outubro, 2014.

[7] DANTAS, M. S. G, OLIVEIRA, E. C. L, DANTAS, T. N. C, NETO, A. A. D, Avaliação antioxidativa de derivados do $\beta$-naftol aplicados a lubrificantes.

http://www.portalabpg.org.br/PDPETRO/4/resumos/4PDPETRO_4_5_0051-1.pdf, Anais do 4PDPETRO, Campinas, SP 4PDPETRO_4_5_0051-1(2007), acessado em 12 de outubro, 2014.

[8] PETROTEST - PetroOXY, - Determination of the Oxidation Stability of Fuels (Gasoline, Diesel, Gasoil, Biodiesel \& FAME,), Grease \& Oil - Ludwig-Erhard-Ring 13 • 15827 Dahlewitz - Germany.

http://www.tqsrl.com/wp-content/uploads/98-1510-Oxidation-Stability-PetroOXY-2.pdf, acessado em 12 de outubro, 2014.

[9] ASTM D6186 - 08, Standard Test Method for Oxidation Induction Time of Lubricating Oils by Pressure Differential Scanning Calorimetry (PDSC). [WWW.astm.org].

[10] OLIVEIRA, F.S.; TEIXEIRA, L.S.G.; ARAÚJO, M.C.U.; M. Korn, Screening analysis to detect adulterations in Brazilian gasoline samples using distillation curves, Fuel, v.83, p.917-923, 2004.

[11] Resolução ANP nº 40/2013 - Especificação da gasolina de uso automotivo. Disponível em: www.anp.gov.br, acessado em 2014.

[12] ASTM D2272, Standard Test Method for Oxidation Stability of Steam Turbine Oils by Rotating Pressure Vessel. [www.astm.org].

[13] ASTM D93, Standard Test Methods for Flash Point By Pensky-Martens Closed Cup Tester [www.astm.org].

[14] SAE International - J300/2013 - Engine Oil Viscosity Classification.

[15] Especificação HANDBOOK - Afton Chemical.

[16] FONSECA, M. M.; Estudo da Gasolina e suas Misturas com Solventes através da Técnica de Análise Térmica. 113f.

[17] CAMPELLO, M. L, Influência do Biodiesel na Estabilidade Termo-Oxidativa do Lubrificante. Dissertação de Mestrado, Universidade Federal do Rio de Janeiro, (2014). 